\title{
Radiation hard monolithic CMOS sensors with small electrodes for HL-LHC and beyond
}

\section{Solans Sanchez, ${ }^{a, *}$ P. Allport, ${ }^{b}$ I. Asensi Tortajada, ${ }^{a, c}$ D. Bortoletto, ${ }^{d}$ C. Buttar, ${ }^{e}$ R. Cardella, ${ }^{a, f}$ F. Dachs, ${ }^{a, g}$ V. Dao, ${ }^{a}$ H. Denizli, ${ }^{h}$ M. Dyndal, ${ }^{a}$ L. Flores Sanz de Acedo, ${ }^{a, e}$ P. Freeman, ${ }^{b}$ A. Gabrielli, ${ }^{a}$ L. Gonella, ${ }^{b}$ K. Oyulmaz, ${ }^{h}$ H. Pernegger, ${ }^{a}$ P. Riedler, ${ }^{a}$ H. Sandaker, ${ }^{f}$ A. Sharma, ${ }^{a}$ W. Snoeys, ${ }^{a}$ J. Torres Pais ${ }^{c}$ and S. Worm ${ }^{i}$}

${ }^{a}$ CERN, Switzerland

${ }^{b}$ University of Birmingham, United Kingdom

${ }^{c}$ Universidad de Valencia, Spain

${ }^{d}$ University of Oxford, United Kingdom

${ }^{e}$ University of Glasgow, United Kingdom

${ }^{f}$ University of Oslo, Norway

${ }^{g}$ University of Vienna, Austria

${ }^{h}$ Bolu Abant Izzet Baysal University, Turkey

${ }^{i}$ DESY (Zeuthen), Germany

E-mail: carlos.solans@cern.ch

The upgrade of tracking detectors for experiments at the HL-LHC and future colliders requires the development of novel radiation hard silicon sensors. We target the replacement of hybrid pixel detectors with Depleted Monolithic Active Pixel Sensors (DMAPS) that are radiation hard monolithic CMOS sensors. We designed, manufactured and tested DMAPS in the TowerJazz $180 \mathrm{~nm}$ CMOS imaging technology with small electrodes pixel designs, that have a pixel pitch well below the current hybrid pixel detectors, and less multiple scattering due to a reduced total silicon thickness. In this document we present the recent results from these sensors manufactured on Czochralski silicon substrates in terms of cluster size, impact on tracking and time resolution from measurements carried out at beam tests on irradiated samples at $1 \mathrm{e} 151 \mathrm{MeV} \mathrm{n} \mathrm{eq}_{\mathrm{eq}} / \mathrm{cm}^{2}$.

40th International Conference on High Energy physics - ICHEP2020

July 28 - August 6, 2020

Prague, Czech Republic (virtual meeting)

\footnotetext{
${ }^{*}$ Speaker
} 


\section{Introduction}

Depleted Monolithic Active Pixel Sensors (DMAPS) have been a very promising technological choice for High Energy Physics experiments in terms of radiation damage, pile-up mitigation, and compatibility with industrial production that can result in an important cost reduction for the experiments at the HL-LHC and beyond. These have been explored in the context of the European Strategy for Particle Physics where the highest priority is to exhaust the physics potential of the LHC that will deliver up to $3000 \mathrm{fb}^{-1}$ after the completion of the Phase-II upgrade during which the inner tracker of ATLAS will be completely replaced by a new one [1].

We focus on DMAPS with small collection electrode and electronics outside the collection well, with small capacitance, high signal to noise ratio, and large drift path, designed in TowerJazz 180 nm CMOS technology with process modification to meet the radiation hardness requirements [2].

\section{The MALTA prototypes}

The MALTA DMAPS is a $22 \times 20 \mathrm{~mm}^{2}$ large demonstrator consisting of $512 \times 512$ pixels of with a pitch of $36.4 \mu^{2}$. It features an asynchronous readout with a clock-less matrix architecture, and 2-3 $\mu \mathrm{m}$ collection electrode size [2,3]. The first prototypes showed poor lateral field after irradiation $[4,5]$ for which additional process modifications were necessary, namely one with an additional extra deep p-well (EDPW) and the other with a gap in the n-layer blanket (NGAP). These were studied on the Mini-MALTA prototype that is a $5 \times 1.7 \mathrm{~mm}^{2}$ DMAPS demonstrator, with $64 \times 16$ pixels of the same size of the MALTA, that showed full efficiency in particle beam tests after $1 \mathrm{e} 15 \mathrm{n}_{\mathrm{eq}} / \mathrm{cm}^{2}$ at 200 electrons threshold and $6 \mathrm{~V}$ bias on sectors with enlarged transistors [6]. Complementary measurements with a focused x-ray beam at Diamond Light Source showed improved charge collection efficiency in the pixel corners with respect to the original process modification [7]. Subsequently, full size MALTA prototypes with the additional process modifications (NGAP and EDPW) were produced on Czochralski substrates, to aim for larger charge collection and higher depletion voltages, and showed almost full efficiency of samples irradiated to $1 \mathrm{e} 15 \mathrm{n}_{\mathrm{eq}} / \mathrm{cm}^{2}$ [8]. In the following we present a subset of these results focused on cluster size, tracking, and time resolution.

\section{Recent results with Czochralski samples}

The data presented in this study were recorded at DESY with an electron beam of $4 \mathrm{GeV}$ converted from bremsstrahlung beams through carbon fibre targets with up to 1000 particles per $\mathrm{cm}^{2}$ energy spread of $5 \%$ and a divergence of $1 \mathrm{mrad}$ [9]. Neutron irradiated samples where irradiated at the Triga nuclear reactor in Slovenia. A dedicated multi-plane read-out system (telescope) based on three $100 \mu m$ thick epitaxial silicon MALTA samples was employed for data acquisition, and reference track reconstruction. General Broken Lines (GBL) algorithm embedded in the Proteus software [10] was used to mitigate the multiple scattering effects of the few-GeV electron beam.

Czochralski $(\mathrm{Cz})$ samples, both with standard process and NGAP modifications, exhibit larger cluster sizes to those produced on epitaxial silicon before irradiation as shown in figure 1 (center and right). Their average values are 1.8 pixels for $\mathrm{Cz}$ standard, 1.4 pixels for $\mathrm{Cz}$ NGAP, and 1.2 
pixels for epitaxial silicon. It should be noted that NGAP has smaller cluster size than the standard modified process. However, since the standard process suffers more from radiation damage, after irradiation, at $1 \mathrm{e} 15 \mathrm{n}_{\mathrm{eq}} / \mathrm{cm}^{2}$ the NGAP has larger cluster size (1.4 vs 1.2 pixels) than standard modified process, as shown in figure 1 (left).
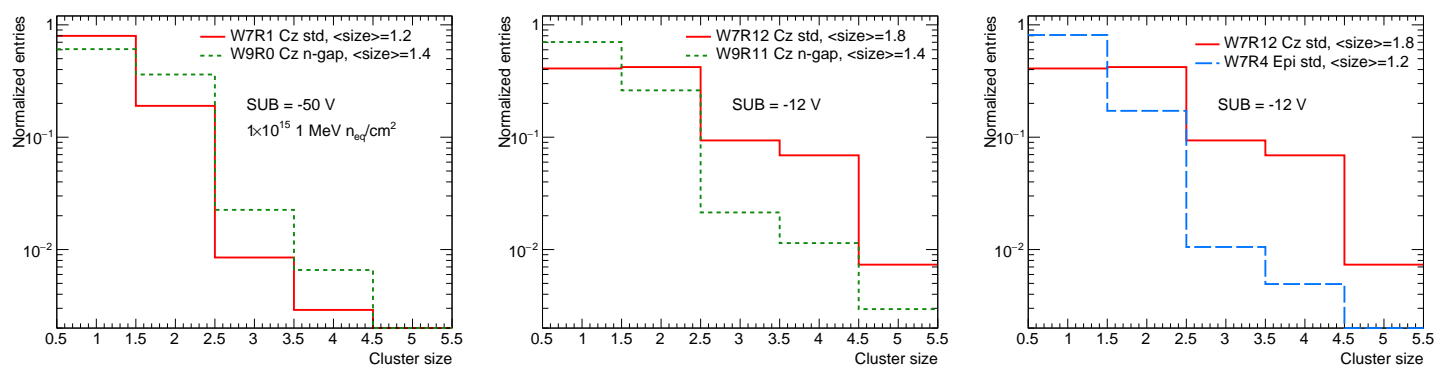

Figure 1: Cluster size distributions of MALTA samples irradiated at $1 \mathrm{e} 15 \mathrm{n}_{\mathrm{eq}} / \mathrm{cm}^{2}$ on Czochralksi substrate with and without NGAP modification at $50 \mathrm{~V}$ bias (left), un-irradiated at $12 \mathrm{~V}$ bias (center), and comparing epitaxial and Czochralski substrates at $12 \mathrm{~V}$ bias (right).
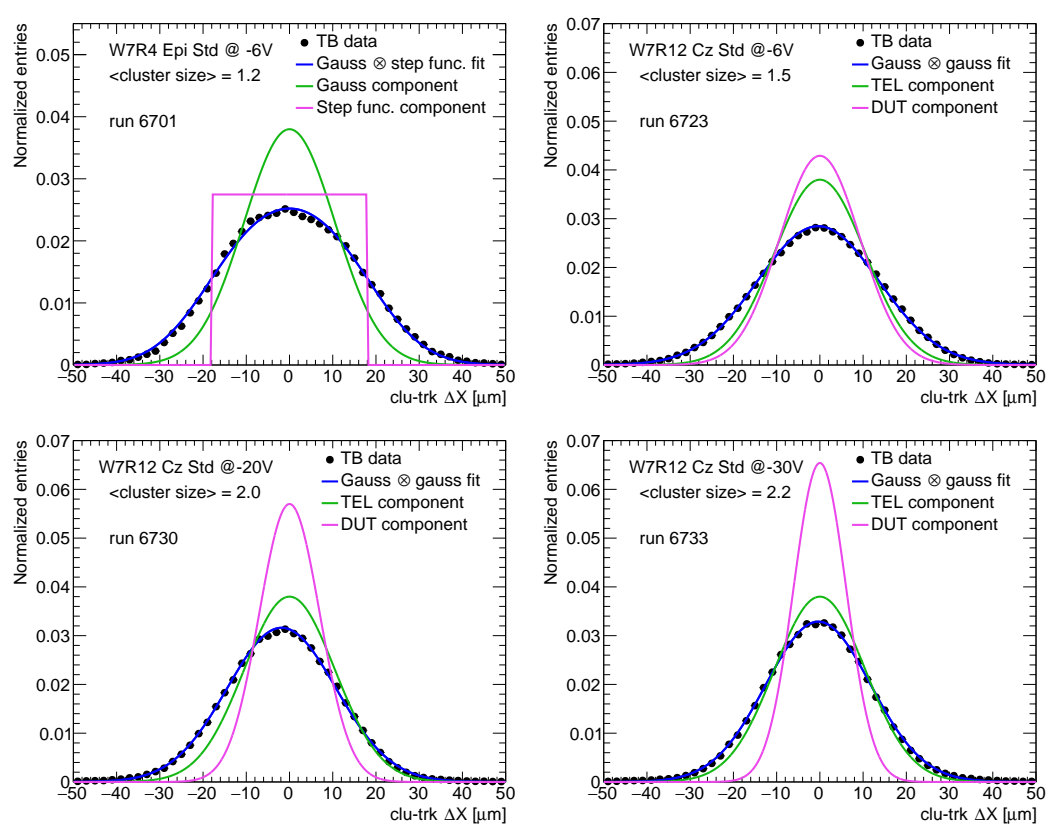

Figure 2: Tracking resolution defined as the distance from the cluster barycenter to the track intercept using a binary clusterizer algorithm on un-irradiated MALTA sample with standard process modification on Czochralski substrate biased to 6, 20 and $30 \mathrm{~V}$, measured with a $4 \mathrm{GeV}$ electron beam at DESY.

Considering the telescope planes are standard epitaxial silicon samples biased to $6 \mathrm{~V}$, the telescope resolution is $10.5 \mu \mathrm{m}$. This value is extracted from the convolution of a Gaussian distribution with a two-sided step function with $36.4 \mu \mathrm{m}$ width corresponding to the pixel pitch of MALTA, as shown on figure 2 (top left). However, when data is fitted to a double Gaussian distribution where the RMS of one of the Gaussians is constrained to $10.5 \mu \mathrm{m}$ compatible with the resolution of the epitaxial based MALTA telescope, the second Gaussian yields a width of 9.4, 
7.0, and $6.1 \mu \mathrm{m}$ for increasing bias voltages of 6,20 , and $30 \mathrm{~V}$, as shown on figure 2 (top right, bottom left and botom right). It would be therefore possible to improve the tracking resolution of the telescope down to $6.1 \mu \mathrm{m}$ by using $\mathrm{Cz}$ samples at higher bias for the reference planes.

Time resolution of the MALTA sensor was evaluated through means of the Pico-TDC ASIC [11]. In this analysis, the reference signal from the three epitaxial silicon MALTA tracking planes and the $\mathrm{Cz}$ MALTA are connected to the Pico-TDC, that is able to measure time difference of the leading edge of the signal from one of the planes used as a reference and the other three planes. This results in three time difference distributions being obtained from events with at least one hit on each plane. The resolution from each distribution is extracted from the core that contains $50 \%$ of the events, and the resolution of the plane of interest is calculated from the combination of the resolution of the time difference distributions given by $\sigma_{2}=\sqrt{\frac{1}{2}\left(\sigma_{12}^{2}+\sigma_{23}^{2}-\sigma_{13}^{2}\right)}$.

Figure 3 shows the time resolution of MALTA sample with standard process modification on Czochralski silicon for different function of bias voltage. For comparison, one point is shown for the same measurement on the epitaxial silicon sample. Time resolution on the epitaxial silicon sample is $2.60 \pm 0.05 \mathrm{~ns}$ at $6 \mathrm{~V}$, while the time resolution on the Czochralski sample is compatible with 1.7 $\pm 0.1 \mathrm{~ns}$ between $10 \mathrm{~V}$ and $30 \mathrm{~V}$. This is compatible with the assumption of a larger electrical field in the Czochralski samples.

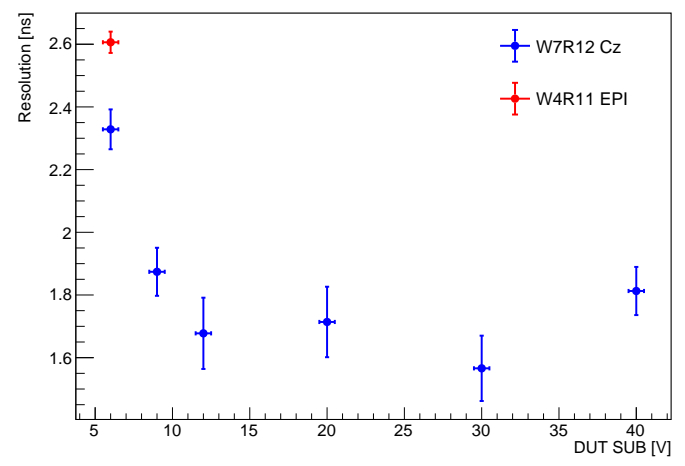

Figure 3: Time resolution comparison of MALTA samples on Czochralski substrate $(\mathrm{Cz})$ with standard process modification and epitaxial silicon (EPI) with gap in n-layer blanket as a function of bias voltage, measured with a $4 \mathrm{GeV}$ electron beam at DESY.

\section{Conclusions and future plans}

MALTA DMAPS produced in $180 \mathrm{~nm}$ TowerJazz technology are an interesting candidate for small electrode monolithic prototypes for HL-LHC and beyond. Additional process modifications (gap in $\mathrm{n}$ - layer and extra deep p-well) showed full efficiency after $1 \mathrm{e} 15 \mathrm{n}_{\mathrm{eq}} / \mathrm{cm}^{2}$ neutron irradiation. Substrate engineering (Czochralski substrates) show increase tracking and timing resolution due to increased depletion depth and charge sharing. At the time of writing a second version of the MALTA demonstrator has been submitted for production, with a smaller matrix, additional process modifications to further address the radiation hardness, and to be manufactured on epitaxial silicon and on Czochralski substrates. This work will continue in the context work package on 
the development of monolithic pixel detectors of the CERN EP department R\&D program in collaboration with other institutes.

\section{Acknowledgements}

This work has been supported by the Marie Sklodowska-Curie Innovative Training Network of the European Commission Horizon 2020 programme under contract number 675587 (STREAM). The measurements leading to these results have been performed at the TestBeam Facility at DESY Hamburg (Germany), a member of the Helmholtz Association (HGF). This project has received funding from the European Union's Horizon 2020 Research and Innovation programme under Grant Agreement number 654168 (IJS, Slovenia).

\section{References}

[1] “Technical Design Report for the ATLAS Inner Tracker Pixel Detector," Tech. Rep. CERNLHCC-2017-021. ATLAS-TDR-030, CERN, Geneva, Sep 2017.

[2] H. Pernegger et al., "First tests of a novel radiation hard CMOS sensor process for Depleted Monolithic Active Pixel Sensors," JINST, vol. 12, p. P06008, 2017.

[3] I. Berdalovic et al., "Monolithic pixel development in TowerJazz $180 \mathrm{~nm}$ CMOS for the outer pixel layers in the ATLAS experiment," JINST, vol. 13, pp. C01023-C01023, jan 2018.

[4] A. Sharma el al., "The MALTA CMOS pixel detector prototype for the ATLAS Pixel ITK," PoS, vol. VERTEX2018, p. 014, 2019.

[5] E.J. Schioppa et al., "Measurement results of the malta monolithic pixel detector," NIM A, vol. 958, p. 162404, 2020. Proceedings of the Vienna Conference on Instrumentation 2019.

[6] M. Dyndal et al., "Mini-MALTA: radiation hard pixel designs for small-electrode monolithic CMOS sensors for the high luminosity LHC," JINST, vol. 15, p. P02005, feb 2020.

[7] M. Mironova et al., "Measurement of the relative response of TowerJazz Mini-MALTA CMOS prototypes at Diamond Light Source," NIM A, vol. 956, p. 163381, 2020.

[8] H. Pernegger et al., "Radiation hard monolithic CMOS sensors with small electrodes for High Luminosity LHC," NIM A, vol. 986, p. 164381, 2021.

[9] R. Diener et al, “The DESY II test beam facility,” NIM A, vol. 922, pp. 265 - 286, 2019.

[10] M. Kiehn, “Proteus beam telescope reconstruction,” Mar. 2019. 10.5281/zenodo.2586736.

[11] L. Perktold and J. Christiansen, "A multichannel time-to-digital converter ASIC with better than 3 ps RMS time resolution," JINST, vol. 9, no. 01, p. C01060, 2014. 